\title{
Analysis the Simulation Performances of EOR Techniques in the Oil Recovery Enhancement
}

\author{
Alireza Baghizadeh ${ }^{1}$, Ali Esfandiari Bayat ${ }^{2}$, Jamal Sheikzakariaee ${ }^{1}$ \\ ${ }^{I}$ Department of Petroleum Engineering, Science and Research Branch, Islamic Azad University, Tehran, Iran. \\ ${ }^{2}$ Department of Reservoir Engineering, Faculty of Petroleum and Chemical Engineering, Science and Research \\ Branch, Islamic Azad University, Tehran, Iran.
}

*Corresponding Author: Ali Esfandiari Bayat, Department of Reservoir Engineering, Faculty of Petroleum and Chemical Engineering, Science and Research Branch, Islamic Azad University, Tehran, Iran.

\begin{abstract}
Regarding the enormous demands for hydrocarbon fossil fuels in numerous industries which enforced Petroleum industries to find novelistic solutions to enhance the oil recovery factor. Due to the reduction of oil volume by the natural depletion mechanisms after the passing of production time, enhanced oil recovery methods are being widely reported in literature and numerous experimental evaluations are operated to address this issue. The objective of this extensive study is to compare the three injectivity scenarios with the natural depletion drive and how to optimize the efficiency of each method during the time period of 3000 days. Consequently, according to the simulation results, foam injection and water injection have played the substantial role in the oil recovery enhancement; in respect of the way, foam injection by the cumulative oil productivity of 88976 barrels per day is the maximum productivity.
\end{abstract}

Keywords: Recovery Factor, Foam Injection, Water Injection, Cumulative Oil Productivity

\section{INTRODUCTION}

Physical and chemical properties of hydrocarbons might vary according to the different reservoir characteristics; in respect of the way, this alteration could divide produced oils into the high API grade (for low viscosity oil) to low API grade (for heavy crude oil with high viscosity).Therefore, the similar extraction methodology could not be used for various types of crude oil, and a set of different methods should be utilized. To achieve the high rate of hydrocarbon, petroleum engineers are always find novel solutions to address this issue. Due to the fact that, the efficiency of primary and secondary techniques in most of the reservoirs are being estimated about $35 \%$ to $50 \%$ of the total initial oil in [17].The parametric mechanisms of natural drives are the primary techniques of hydrocarbon production and by passing the well lifelong pressure drop, the importance of enhanced oil recovery methodologies are urgently required for the reservoirs. Hence, enhanced oil recovery techniques are entailed; Improved water Flooding, Polymer Flooding, Microbial injection, Gas Flooding, Enriched gas injection, Light gas injection, Chemical Flooding, Nitrogen gas flooding, Carbon dioxide flooding, Miscible flooding, Thermal recovery, Steam Flooding and, Steam cycle Injection [8-12]. Chemical Flooding are being divided into two principal categories, Surfactant Flooding and Foam Flooding. Surfactant is a simple substance and only a small amount of it, would reduce the water surface tension. Surfactants are usually organic materials that contain hydrophobic (water repellant)and hydrophilic groups (water absorbent) which they are soluble in organic solvents and water soluble with the molecular structure and it subsequently reduce the surface tension and wateroil and oil-water interface. It also reduces the drainage of the water and oil interface by the liquidliquid interface. A large number of surfactant molecules can be coupled together in a soluble mass and form a mass called Micelle which is clearly shown in figure 1 [13-17].

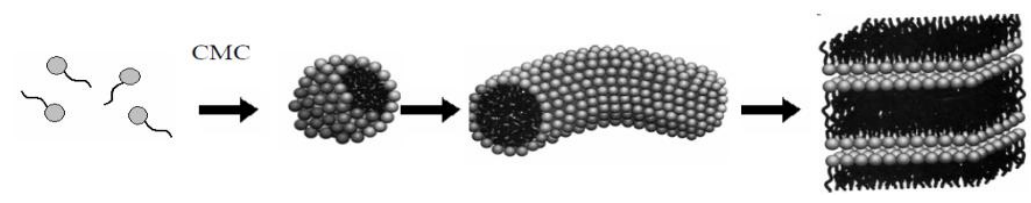

Figure1. Surfactant and Micelle Forming 
The foam behavior in the porous medium utterly depends on the continuity of the environment and the geometry of the environment, that is to say that, the principal characteristics of porous medium in the reservoir would play a substantial role in the foam flowing through the reservoir. These principal factors are;

- The distribution size of continuous porous spaces throughout the porous medium; the foam production mechanism and the foam loss depend heavily on the size of the environment in which it could move.

- It should be taken into account that porous spaces are not entirely spherical and are somewhat angular; for larger empty spaces, thinner fluids are placed in the empty spaces covered by the gas and cover the blank spaces as thin film walls. Non-trapping phase is located in the center of vacant space. Moreover, smaller vacancies are also completely occupied by the flowing agent.

- When the flow rate is very low and the capillary forces are low, the capillary pressure is determined by the phase saturation of the thrust phase and the amount of superficial fluidity. During fuzzy flow, the non-trapping fluid moves through the cracks of empty spaces. The thinner fluids in the small channels are interconnected and flow through the corners of empty spaces where non-tributary fluids are present [18-23].

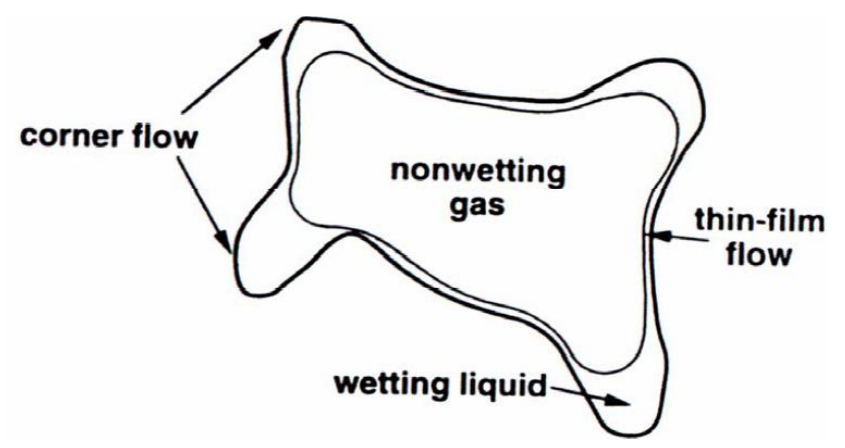

Figure2. Empty spaces corners in the porous medium

Foam stability can be explained by examining the film of lamella and gas bubbles. Since foam stability is strongly dependent on the stability of fluid films and the rupture of these films, it is essential to know the foam stability and foam strength mechanisms to check foam stability. In the lamella, the polar groups have been directed to the surfactant into the film. This is clearly depicted in figure 3. In order to determine the range of membrane stability in foam, it is important to dilute and interconnect it.

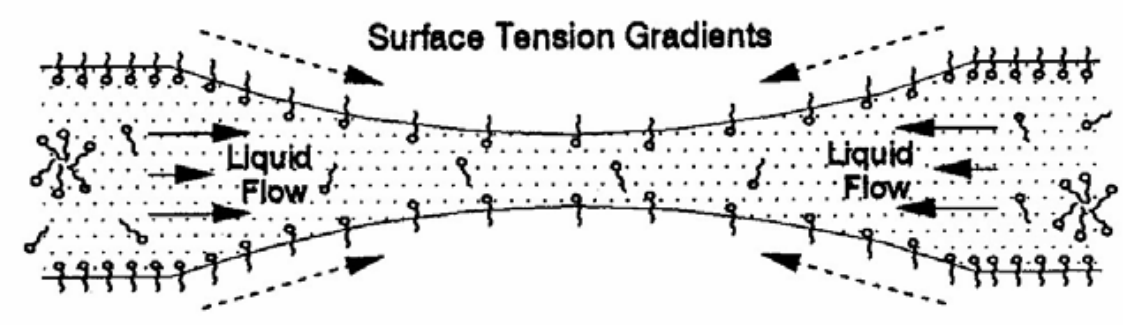

Figure3. Formation of Foam

Simujo (2011) conducted a laboratory analysis titled "Incompatible Foam Injection for Growth Exposure" to determine the behavior of foam and gas incompatible in sandstone cores. These results can also be used for other processes associated with oil extraction (for example, blocking of high permeability layers, mobility control in gas injection processes). Furthermore, a systematic study was carried out on the behavior of foam in the Balkan environment in order to investigate the effect of oil on the ability to foam. The effect of surfactant concentration and injection rate on the amount of foam mobility and also on the rate of foam release in the porous medium was investigated. Foam behavior in bulk mode is the behavior of a set of commercial surfactants used in the industry was investigated through testing (foam column testing) [24-27]. The study of the stability behavior of the column of foam formed in the presence of oil showed that the height of the foam AOS decreases in two steps; 
1) A rapid initial reduction in the height of the foam column at the initial time, which eventually caused the foam column to reach a constant height

2) A secondary reduction due to the fact that the height of the residual foam column decreased steadily (albeit with a lower reduction rate than the initial state).

\section{Methodology}

There are two mechanistic models that describe the flow of foam in the porous medium; bubble immigration model and breakingmodification model.

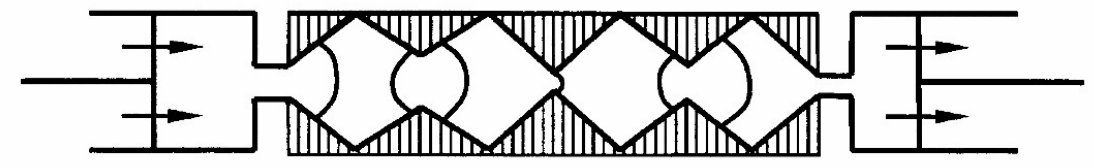

Figure4.The flow of bubble transfers in a porousmedium

Bubble immigration model was being stated by the false (1986) for the first time. In this theory, the gas flow throughout the bubbles were extracted by the lamella process; in respect of the way, gas droplets are flowed in the length porous medium in a long chain. On the contrary, in the breaking modification model, there are not any specific bubbles that would be able to mobilize in the long pathway. Hence, gas droplets during the porous medium are eliminated and reproduced again. Moreover, liquid flow is occurred in the continuous network of bubbles.

The enhanced recovery techniques with the utilization of foam should be accordance of appropriate circumstances of the reservoir in order to stabilize the foam and proper mobilization of oil should be taken into account. Foam injection has significantly affected by the several parameters such as oil saturation, water saturation which is directly affect the capillary pressure, oil composition, reservoir heterogeneity and, capillary number which is the illustration of flow rates. Among these crucial parameters, the considerable influence of water saturation, oil saturation and, surfactant saturation is being widely experimented in the literature. Furthermore, it should be noted that the profound impact of foam might be vanished due to the high oil saturation and low water saturation (high capillary pressure). The sufficient presence of surfactant through the reservoir is a key parameter in the formation of foam. Thereby, if each of mentioned discrepancies in the formation of foam caused to the virtually elimination of foam in the reservoir and subsequently the injected gas is penetrated to the injection well. Therefore, the less oil volume is mobilized forward to the wellbore surficial equipment. To avoid this phenomenon, intelligent designation and appropriate simulation of foam injection should be essentially considered in the procedure of foam flooding. Due to the fact that, the proper selection of surfactant should be economically prospective and it subsequently optimize the current recovery factor.

\subsection{Field Description}

The properties of simulated reservoir which is studied in this modeling consists of $15 \times 15 \times 15$ blocks which is schematically plotted in figure 5 . The size of each block for the shale layer is $100 * 100 * 1$ and for the reservoir layer is $100 * 100 * 100$ feet, which results in a cubic reservoir of 1,500 feet and a height of 301 feet. The reservoir has two sandstone layers which each of them is separated by a nonpermeable shale layer. Thus, there is no flow regime between the layers in the reservoir layers.

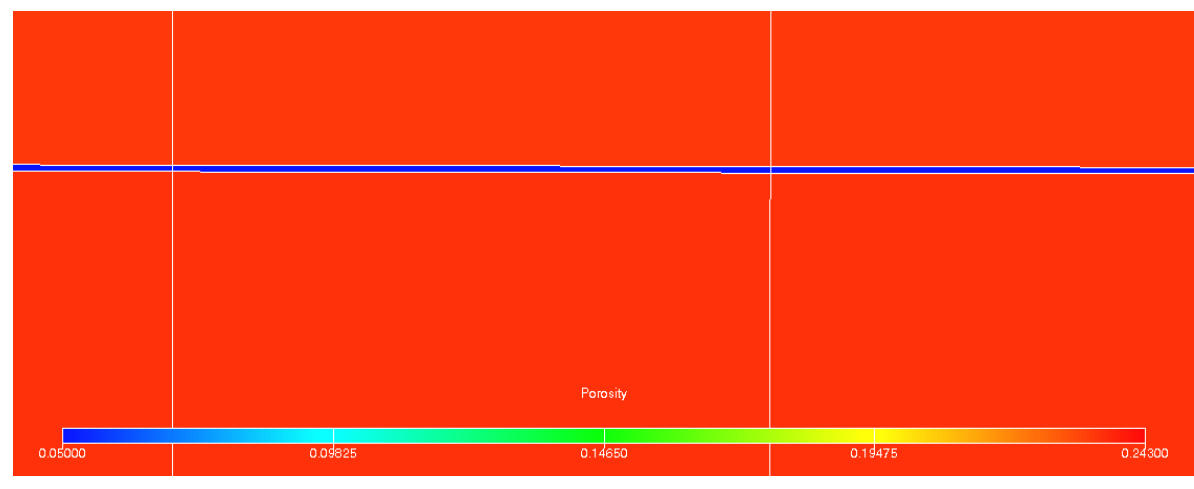

Figure5. Status of Shale Layers between the second and third layer of the reservoir 


\section{RESULTS AND DISCUSSION}

In this comprehensive study, three injection scenarios are being compared with the natural depletion mechanisms. As can be seen in table 1, it is a common sense that production rate according to the natural depletion mechanisms has been declined by passing the time; in respect of the way, in the 3090 days of production, any oil volume from these mechanisms are produced and the significant role of enhanced recovery techniques are being seen in this stage. As can be seen in figure 6 , the effect of natural depletion mechanisms on the production rate decreased gradually with the time increase.

Table1. Comparison of different injection scenarios with the Natural Depletion Drive

\begin{tabular}{|c|c|c|c|c|}
\hline Time (Days) & $\begin{array}{c}\text { Foam Injection } \\
\text { (STB/Day) }\end{array}$ & $\begin{array}{c}\text { Gas Injection } \\
\text { (STB/Day) }\end{array}$ & $\begin{array}{c}\text { Natural Depletion } \\
\text { (STB/Day) }\end{array}$ & $\begin{array}{c}\begin{array}{c}\text { Water Injection } \\
\text { (STB/Day) }\end{array} \\
\end{array}$ \\
\hline 30 & 981 & 979 & 980 & 980 \\
\hline 60 & 977 & 971 & 973 & 973 \\
\hline 90 & 974 & 964 & 965 & 967 \\
\hline 630 & 976 & 905 & 867 & 902 \\
\hline 660 & 972 & 904 & 863 & 899 \\
\hline 690 & 968 & 903 & 860 & 895 \\
\hline 720 & 963 & 902 & 857 & 892 \\
\hline 870 & 924 & 905 & 845 & 870 \\
\hline 900 & 914 & 907 & 844 & 865 \\
\hline 930 & 904 & 907 & 842 & 860 \\
\hline 1470 & 759 & 898 & 798 & 772 \\
\hline 1500 & 753 & 897 & 796 & 767 \\
\hline 1530 & 747 & 895 & 791 & 760 \\
\hline 1560 & 742 & 893 & 786 & 754 \\
\hline 1440 & 764 & 900 & 802 & 777 \\
\hline 1470 & 759 & 898 & 798 & 772 \\
\hline 1500 & 753 & 897 & 796 & 767 \\
\hline 2310 & 633 & 706 & 40 & 641 \\
\hline 2340 & 629 & 682 & 33 & 637 \\
\hline 2370 & 626 & 653 & 28 & 633 \\
\hline 2520 & 611 & 173 & 13 & 616 \\
\hline 2550 & 608 & 134 & 11 & 612 \\
\hline 2580 & 606 & 105 & 9 & 609 \\
\hline 2610 & 603 & 85 & 8 & 605 \\
\hline 2640 & 600 & 69 & 7 & 602 \\
\hline 2670 & 598 & 58 & 6 & 598 \\
\hline 2700 & 595 & 51 & 5 & 595 \\
\hline 2880 & 582 & 35 & 2 & 577 \\
\hline 2910 & 580 & 34 & 1 & 574 \\
\hline 3060 & 570 & 34 & 1 & 558 \\
\hline 3090 & 568 & 34 & 0 & 555 \\
\hline 3120 & 566 & 34 & 0 & 552 \\
\hline 3150 & 564 & 34 & 0 & 549 \\
\hline
\end{tabular}

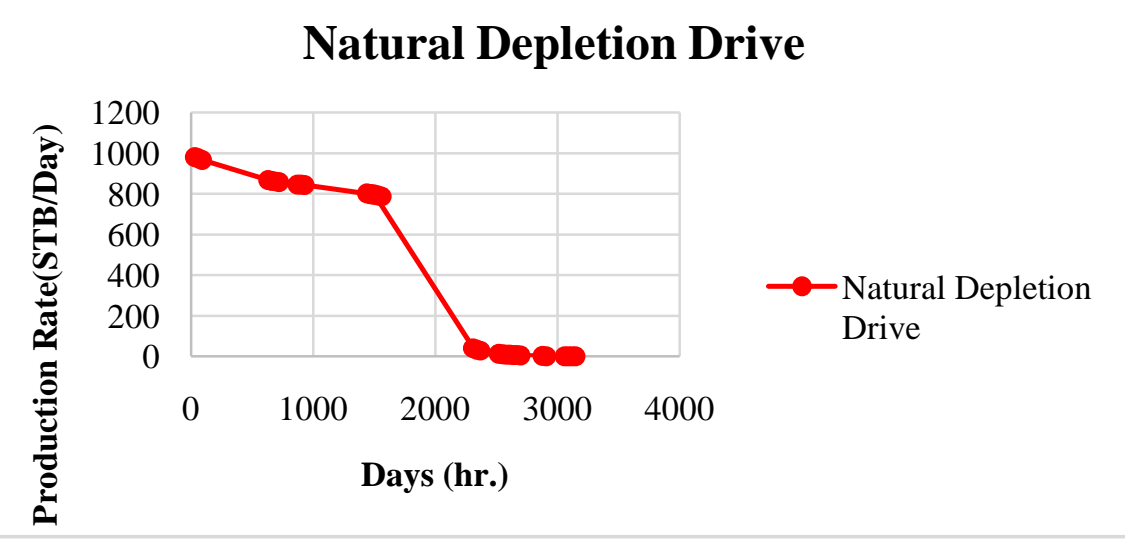

Figure6. Effect of Natural Depletion Drive on the Production rate 
As can be seen in figure 7, the injection of foam through the reservoir caused to stabilize the bottom hole pressure and subsequently the production rate after the 3000 days of production is about 600 STB/ Day.

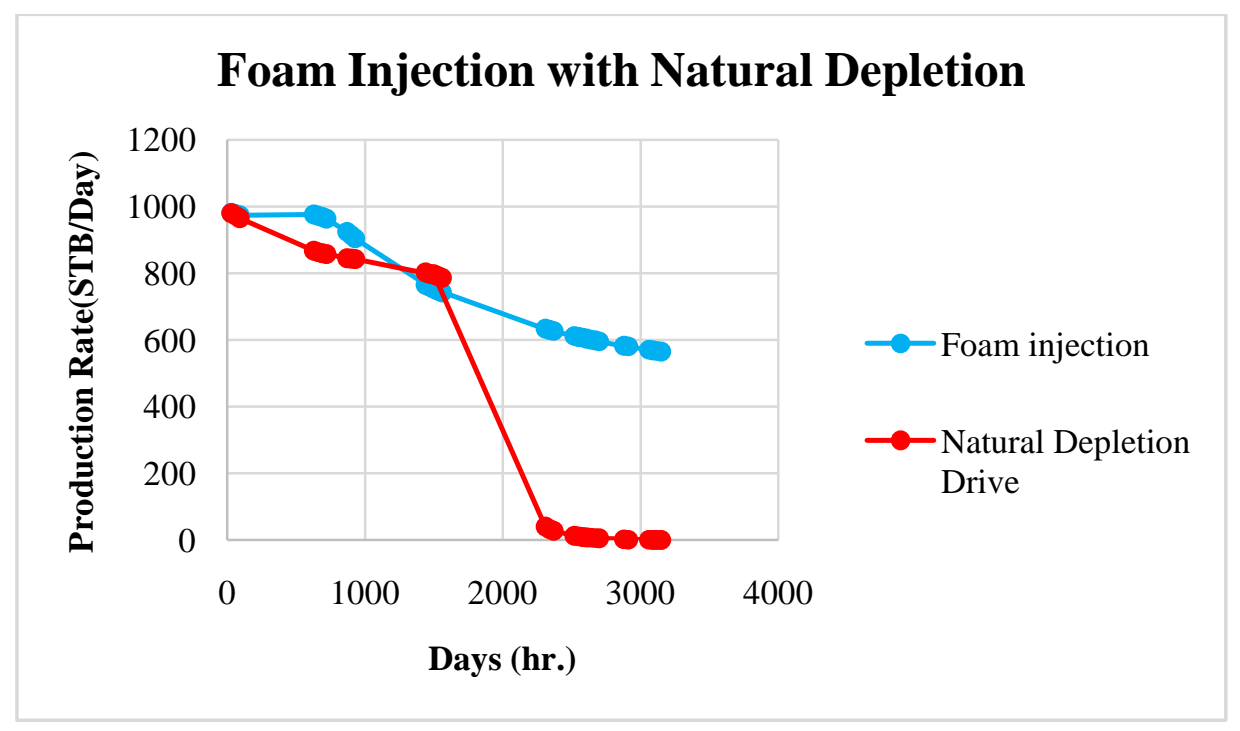

Figure7. Effect of Natural Depletion Drive and Foam injection on the Production rate

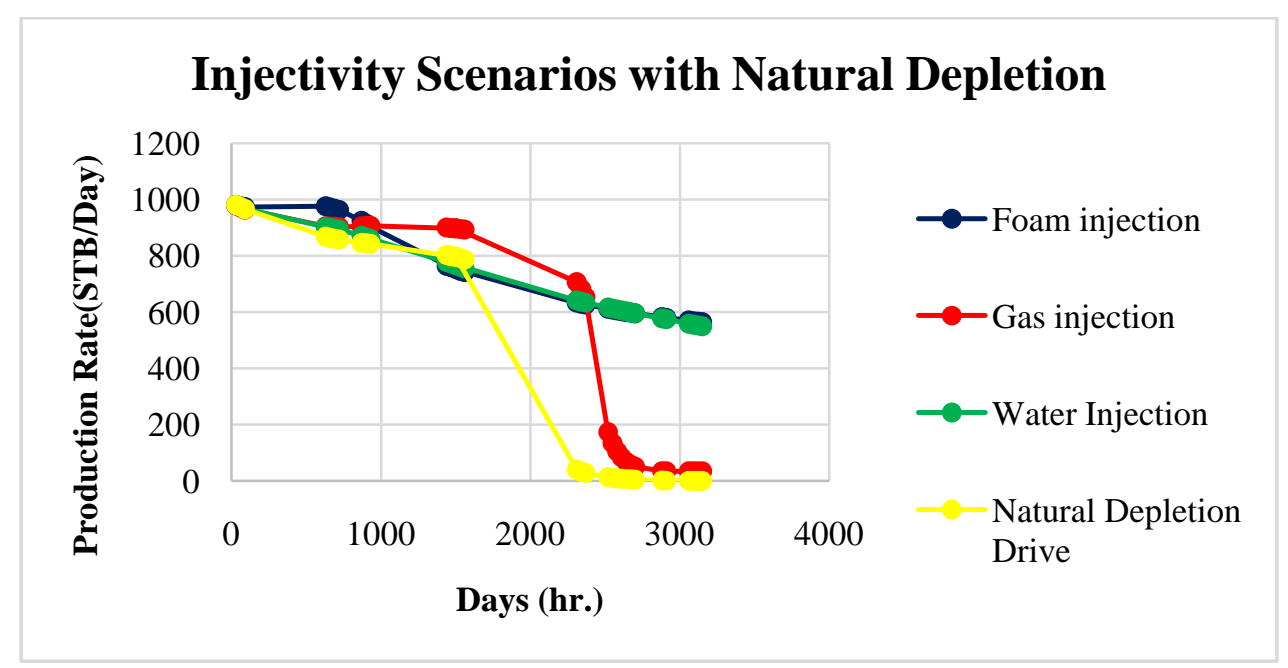

Figure8. Effect of Natural Depletion Drive and three injection scenarios on the Production rate

As can be seen in figure 8, foam flooding and water injection scenario have the maximum production rate after the 3000 days of production and subsequently, it indicates that these two injection scenarios are the best scenarios for producing oil rather than gas injection. Moreover, the cumulative oil production volume regarding the injection of foam in the reservoir after passing the simulation time is about 88,975 barrels, which is the highest production rate among other simulation methods are assigned to themselves. Since then, water injection is in the next stage of cumulative recovery. The cumulative oil production is statistically depicted in table 2 .

Table2. Cumulative Oil Production for each scenario

\begin{tabular}{|c|c|c|c|c|}
\hline EOR Technique & $\begin{array}{c}\text { N/A (Natural } \\
\text { Depletion) }\end{array}$ & $\begin{array}{c}\text { Nitrogen Foam } \\
\text { Injection }\end{array}$ & $\begin{array}{c}\text { Gas } \\
\text { Injection }\end{array}$ & $\begin{array}{c}\text { Water } \\
\text { Injection }\end{array}$ \\
\hline $\begin{array}{c}\text { Cumulative Oil Prod. } \\
\text { (STB/Day) }\end{array}$ & 55567 & 88976 & 72898 & 87058 \\
\hline
\end{tabular}

\section{SUgGESTION FOR FUTURE RESEARCH}

- Evaluation the performance of nitrogen foam and its comparison with other injection methods.

- Investigate and sensitize various parameters of foam injection and achieve an optimal method in areas where use of nitrogen foam seems appropriate. 
- Laboratory studies and corrosive flooding with nitrogen foam for core samples from the country's fields and achieving foam injection parameters and their use in simulation.

\section{CONCLUSiON}

Enhanced oil recovery techniques regarding the numerous administration of hydrocarbon fossil fuels are being popularly utilized in the petroleum industries and hence, engineers try to find the proper techniques with the least economically expenses. In this comprehensive study, a sandstone reservoir with the underground aquifer, the foam injectivity scenario caused to reduce the water volume significantly, enhance the oil productivity and, subsequently maintain the reservoir bottom hole pressure. On the other hand, due to the lifelong of a well for the injectivity of foam in the simulation time period of 3000 days, it would be a preferable method for enhancing the oil recovery.

\section{REFERENCES}

[1] Z. Zhang, V. L. Freedman, and L. Zhong, "Foam Transport in Porous Media: A Review: Pacific Northwest National Laboratory", 2009.

[2] M. Namdar Zanganeh, "Simulation and optimization of foam EOR processes," TU Delft PhD (July 2011), 2011.

[3] R. Farajzadeh, A. Andrianov, and P. Zitha, "Investigation of immiscible and miscible foam for enhancing oil recovery," Industrial \& Engineering Chemistry Research, vol. 49, pp. 1910-1919, 2009.

[4] M. Simjoo, "Immiscible foam for enhancing oil recovery," Petroleum Engineering, p. 187, 2012.

[5] R. Farajzadeh, A. Andrianov, and P. L. J. Zitha, "Foam assisted oil recovery at miscible and immiscible conditions," in Kuwait International Petroleum Conference and Exhibition, 2009.

[6] A. Andrianov, M. Liu, and W. Rossen, "Sweep Efficiency in CO2 Foam Simulations with Oil (SPE 142999)," in 73rd EAGE Conference \& Exhibition, 2011.

[7] Rabbani, E., Davarpanah, A., Memariani, M. An Experimental Study of Acidizing Operation Performances on the Wellbore Productivity Index Enhancement. Journal of Petroleum Exploration and Production Technology, doi.org/ 10.1007/s13202-018-0441-8, 2018.

[8] L. L. Schramm, E. N. Stasiuk, and D. G. Marangoni, "2 Surfactants and their applications," Annual Reports Section" C"(Physical Chemistry), vol. 99, pp. 3-48, 2003.

[9] L. L. Schramm, "Surfactants: fundamentals and applications in the petroleum industry": Cambridge University Press, 2000.

[10] W. Yan, "Foam for Mobility Control on Alkaline/Surfactant/Enhanced Oil Recovery Process," Doctoral Thesis, Rice University. http://hdl. handle. net/1911/18997, 2006.

[11] A. K. Vikingstad, "Static and dynamic studies of foam and foam-oil interactions," 2006.

[12] T. Dicksen, G. J. Hirasaki, and C. A. Miller, "Conditions for foam generation in homogeneous porous media," in SPE/DOE Improved Oil Recovery Symposium, 2002.

[13] G. Hirasaki, C. A. Miller, and M. Puerto, "Recent advances in surfactant EOR," SPE Journal, vol. 16, pp. 889-907, 2011.

[14] D. Shan and W. Rossen, "Optimal Injection Strategies for Foam IOR. SPEJ 9 (2): 132-150," SPE-75180PA. DOI: $10.2118 / 75180-P A 2004$.

[15] W. R. Rossen and W. J. Renkema, "Success of Foam SAG Processes in Heterogeneous Reservoirs," in SPE Annual Technical Conference and Exhibition, 2007.

[16] Davarpanah, A., Mirshekari, B., Behbahani, T. J., \& Hemmati, M. Integrated production logging tools approach for convenient experimental individual layer permeability measurements in a multi-layered fractured reservoir. Journal of Petroleum Exploration and Production Technology, 1-9 doi.org/10.1007/s13202-017-0422-3, 2018.

[17] S. I. Kam, Q. P. Nguyen, Q. Li, and W. R. Rossen, "Dynamic simulations with an improved model for foam generation," SPE Journal, vol. 12, pp. 35-48, 2007.

[18] R. Farajzadeh, A. Andrianov, R. Krastev, W. Rossen, and G. Hirasaki, "Foam-Oil Interaction in Porous Media-Implications for Foam-assisted Enhanced Oil Recovery (SPE 154197)," in 74th EAGE Conference \& Exhibition, 2012.

[19] L. Cheng, A. Reme, D. Shan, D. Coombe, and W. Rossen, "Simulating foam processes at high and low foam qualities," in SPE/DOE Improved Oil Recovery Symposium, 2000.

[20] W. R. Rossen, "Numerical challenges in foam simulation: a review," in SPE Annual Technical Conference and Exhibition, 2013. 
[21] S. H. Talebian, P. Rahim Masoudi, I. M. Tan, and P. L. Zitha, "Foam assisted CO2-EOR; Concepts, Challenges and Applications," 2013.

[22] M. Simjoo, Y. Dong, A. Andrianov, M. Talanana, and P. L. Zitha, "Novel insight into foam mobility control," in International Petroleum Technology Conference, 2011.

[23] M. Simjoo, T. Rezaei, A. Andrianov, and P. Zitha, "Foam stability in the presence of oil: Effect of surfactant concentration and oil type," Colloids and Surfaces A: Physicochemical and Engineering Aspects, vol. 438, pp. 148-158, 2013.

[24] M. Sohrabi and S. A. Farzaneh, "A Review of the Status of Foam Application in Enhanced Oil Recovery," in EAGE Annual Conference \& Exhibition incorporating SPE Europec, 2013.

[25] R. M. de Velde Harsenhorst, A. S. Dharma, A. Andrianov, and W. R. Rossen, "Extension and Verification of a Simple Model for Vertical Sweep in Foam Surfactant-Alternating-Gas Displacements," SPE Reservoir Evaluation \& Engineering, 2014.

[26] Davarpanah, A., \& Nassabeh, M. M. Optimization of drilling parameters by analysis of formation strength properties with using mechanical specific energy. Bulgarian Chemical Communications Journal, Special Issue J, (pp. $364-375), 2017$.

[27] Davarpanah, A., \& Nassabeh, M. M. Recommendations for Optimizing the Efficiency of Polymer Flooding Techniques in Production Operation of an Oilfield. Electronic Journal of Biology, 13(3), 2017

\section{AUTHORS' BIOGRAPHY}
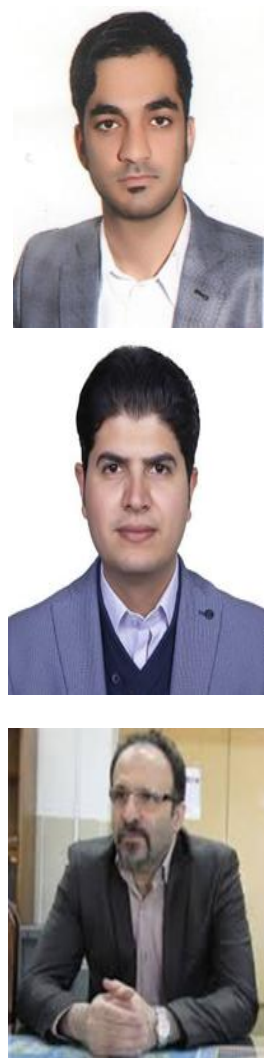

Alireza Baghizadeh, Master degree of Petroleum Engineering at Islamic Azad University, Tehran science and research Branch. My current interests in petroleum engineering sciences include a full-field study of Data Gathering, Reservoir Simulation, History Matching and Prediction.

Dr. Ali Esfandiari Bayat, Post Doctorate Degree of Petroleum Engineering and he is assistant professor and M.SC. and doctoral student supervisor at Department of Reservoir Engineering, Faculty of Petroleum and Chemical Engineering, Science and Research Branch, Islamic Azad University, Tehran, Iran

Dr. Jamal Sheikhzakariaee, is associate professor and M.SC. and doctoral student supervisor, and Deputy of Petroleum and Chemical Engineering Campus at Islamic Azad University, Tehran science and research Branch, where he specializes in geological engineering and operates such related projects in the university.

Citation: A. Baghizadeh et.al, (2018). Analysis the Simulation Performances of EOR Techniques in the Oil Recovery Enhancement, International Journal of Petroleum and Petrochemical Engineering (IJPPE), 4(2), pp.2228, DOI: http://dx.doi.org/10.20431/2454-7980.0402004

Copyright: (c) 2018 A. Baghizadeh. This is an open-access article distributed under the terms of the Creative Commons Attribution License, which permits unrestricted use, distribution, and reproduction in any medium, provided the original author and source are credited 